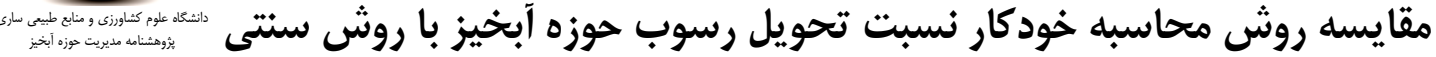

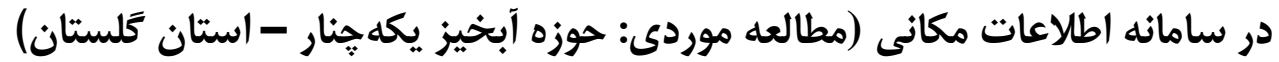

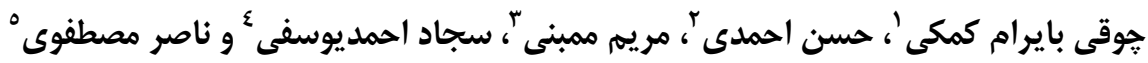

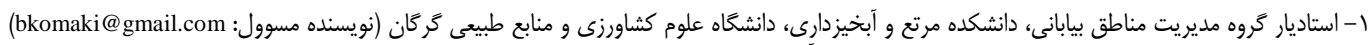

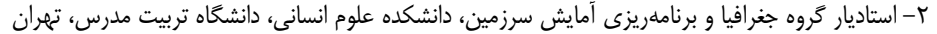

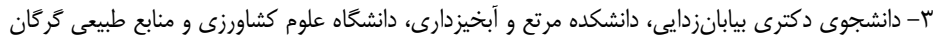

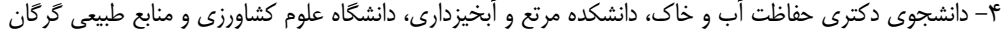

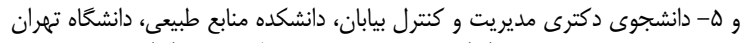

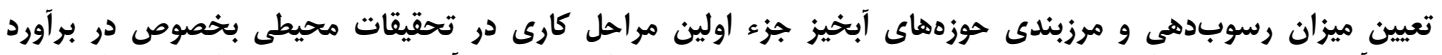

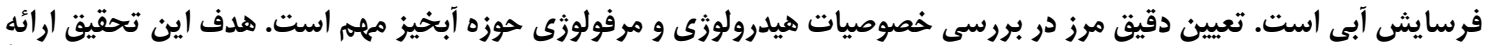

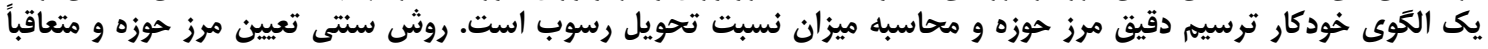

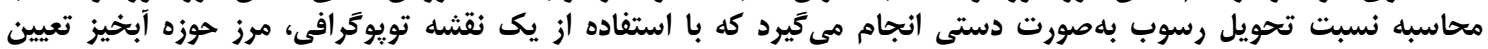

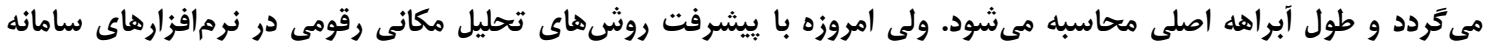

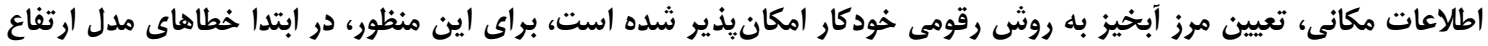

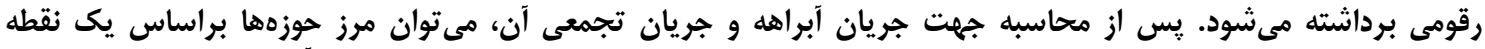

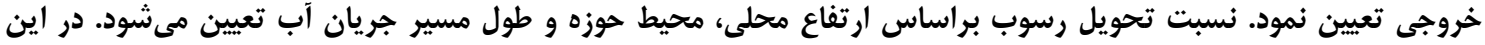

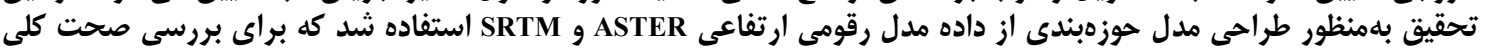

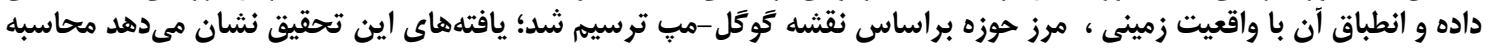

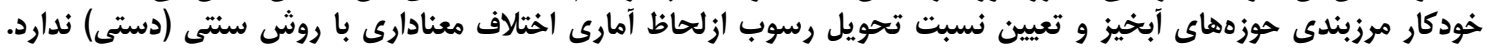

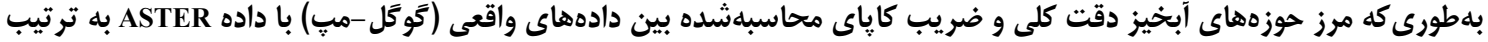

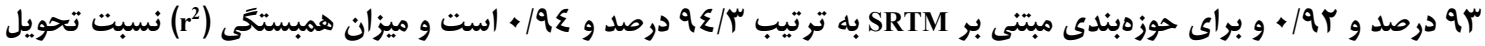

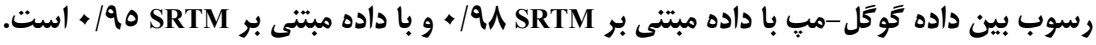

وازههاى كليدى: مرزبندى، قطعلبندى، مدل رقومى ارتفاع، نسبت تحويل رسوب، رود اترك.

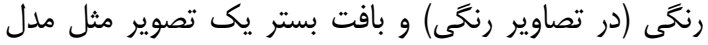

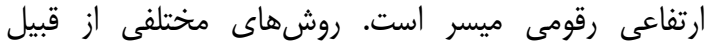

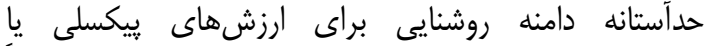

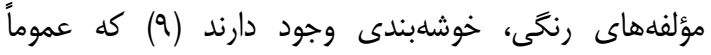
براساس خصوصيات نقطهاى تعريف مى خرّردند. البته با استفاده

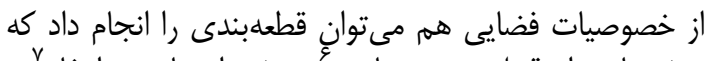

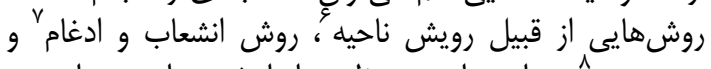

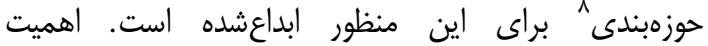

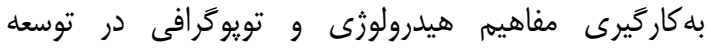
روشهاى قطعلبندى به اثبات رسيده است. درئ در اين روشي،

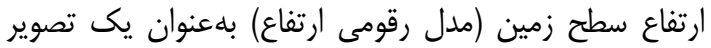

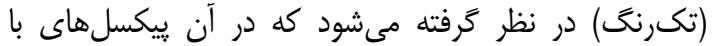

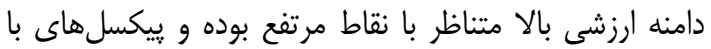

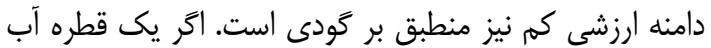

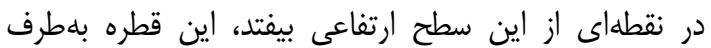

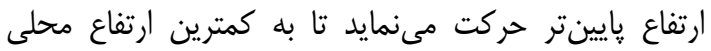

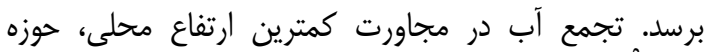

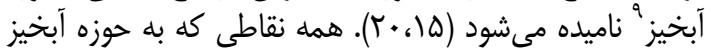

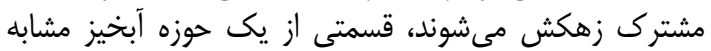

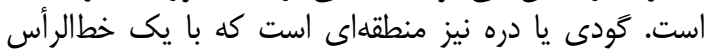

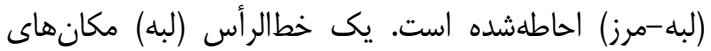

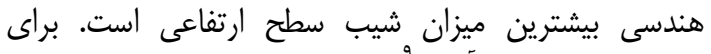
محاسبه حوزمبندى آبخيز" در يك تصوير رسترى روشهاى ارتفاع إي

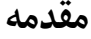

ترسيم مرز حوزه آبخيز بلهصورت دستى با استفاده از

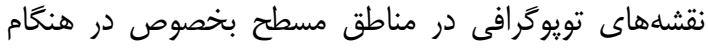

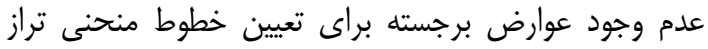

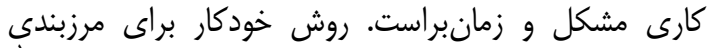

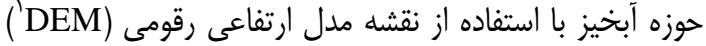

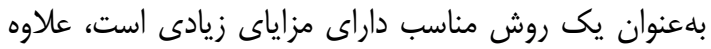

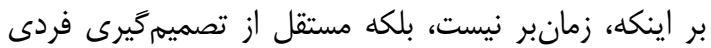

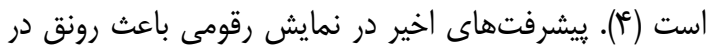

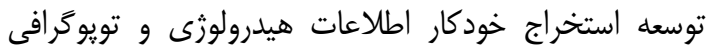

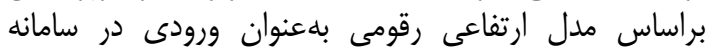

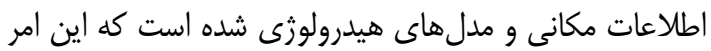

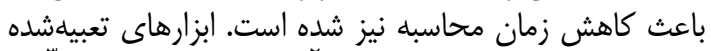

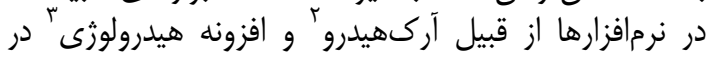

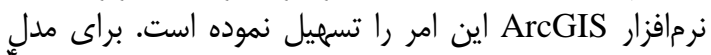

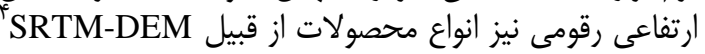

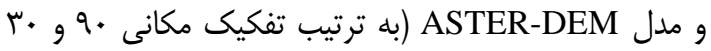

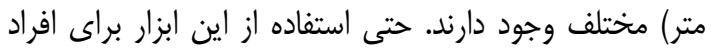

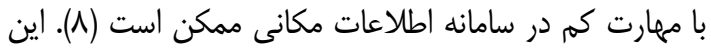

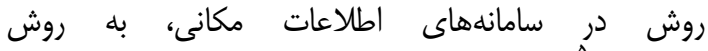
قطعهبندى ه تصوير صورت مى گيرد. قطعلبندى تصوير مستلزم

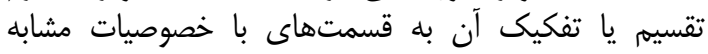

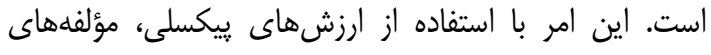

1- Digital Elevation Model

4- Shuttle Radar Topography Mission

7- Split and Merge
3- Hydrology Tool

6- Region Growing

9- Watershed Delineation 
هموار، نتايج آن رضايتبخش نيست. شناسايى دقيق مرز

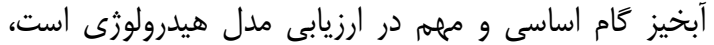

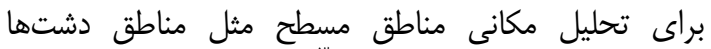

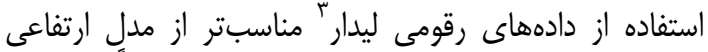

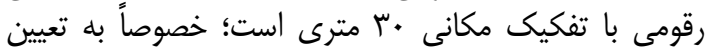

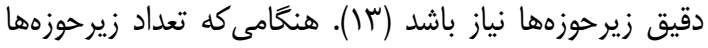

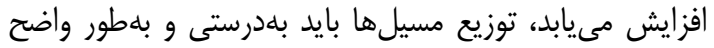

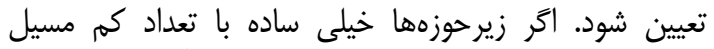

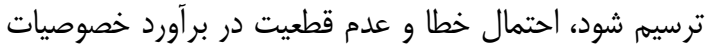

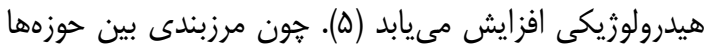

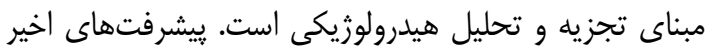

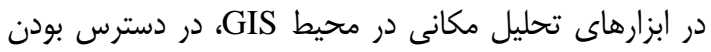

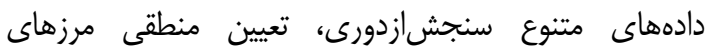

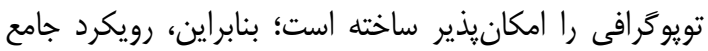

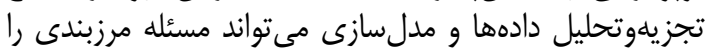

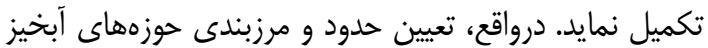

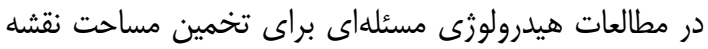

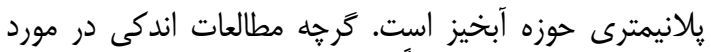

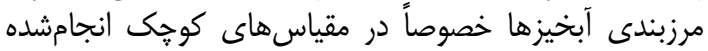

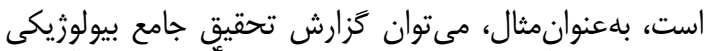
و كشاورزى آمريكا در زيرحوزه ايلامانكا" در شمال هال آمريكا

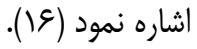

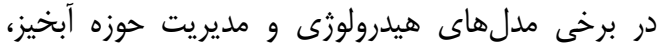

خصوصيات حوزه آبخيز براى تعيين ميزان فرسي فرايش و وانتقال

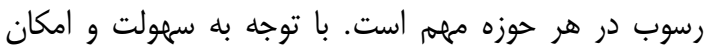

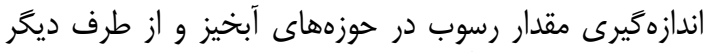

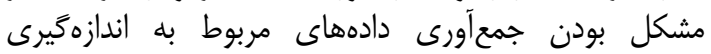

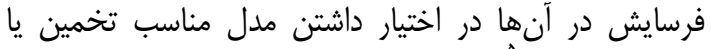

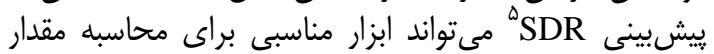

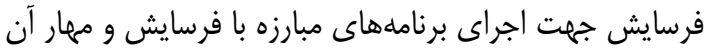

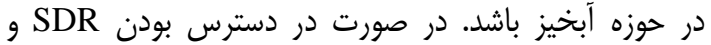

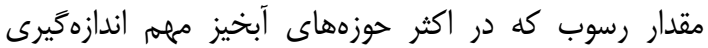

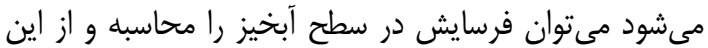

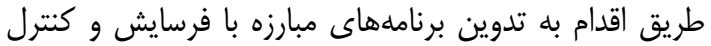

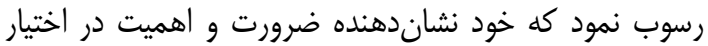

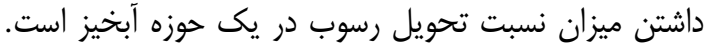
تاكنون هيج رابطه يا مدل مشخصى بران براى برآورد

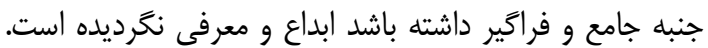

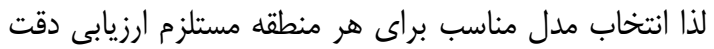

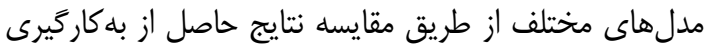

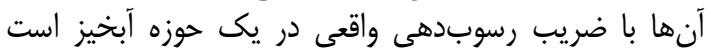

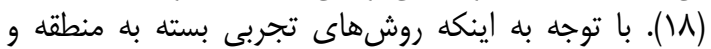

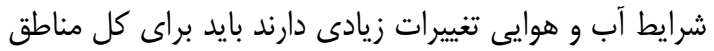

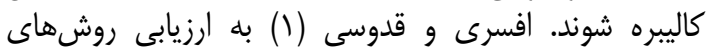

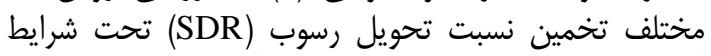

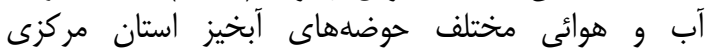

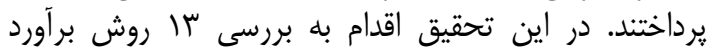

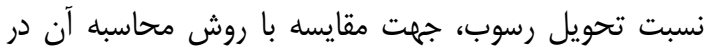
مدل EPM جهت ارتقاء دقت مدل در دو حوضه آبخيز خمين آن درش
بارش و سيل وجود دارد؛ در روش محاسبه حوزه آبخيز بر

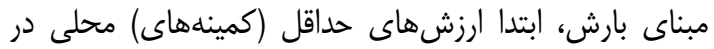

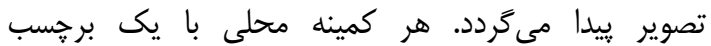

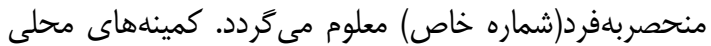

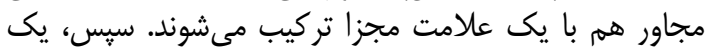

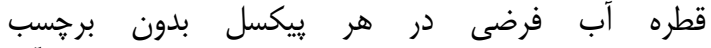

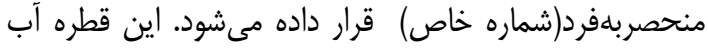

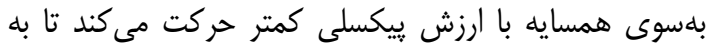

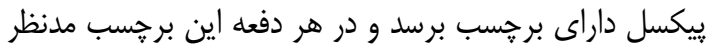

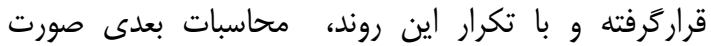

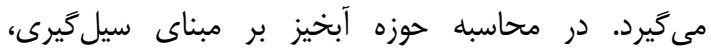

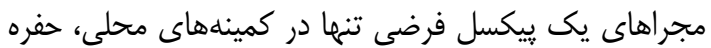

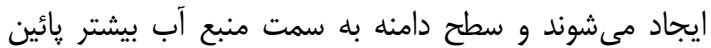

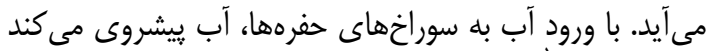

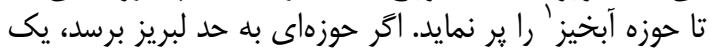

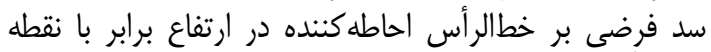

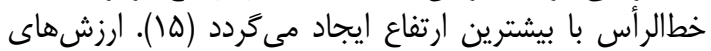

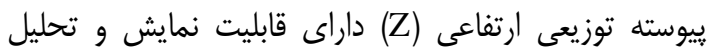

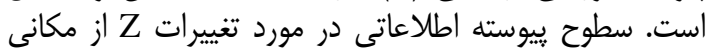

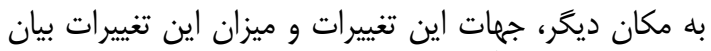

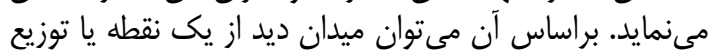

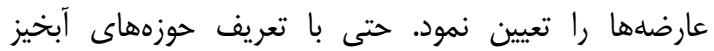

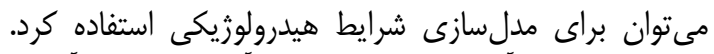

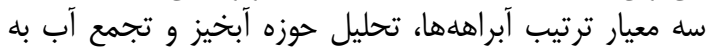

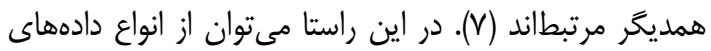

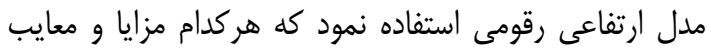

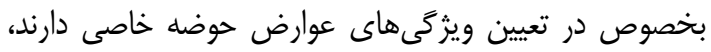

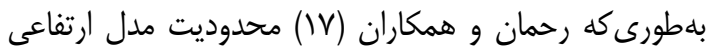

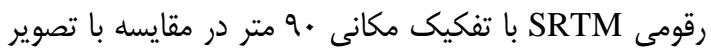

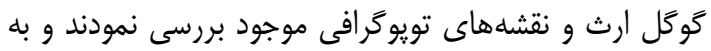

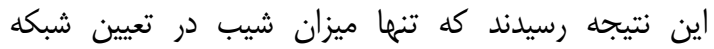

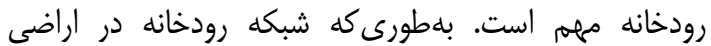

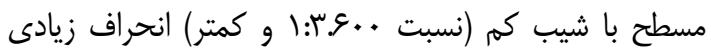

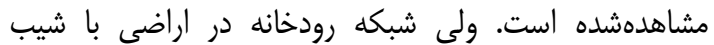

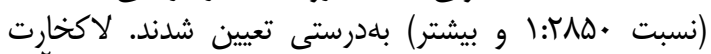

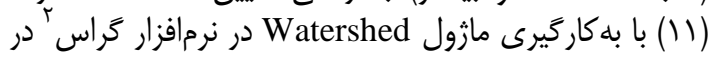

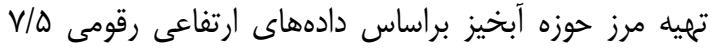

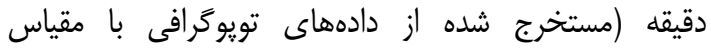

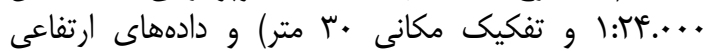

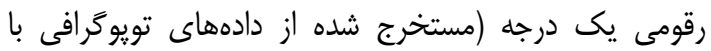

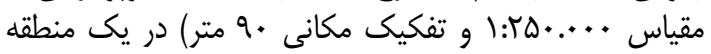

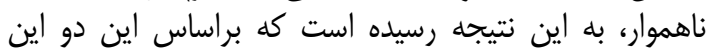

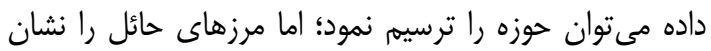

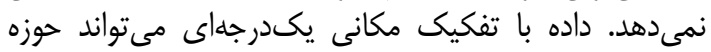

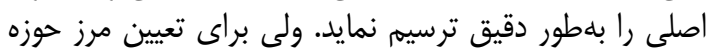

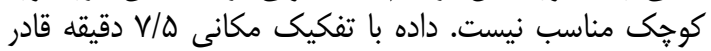

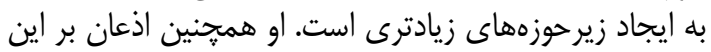

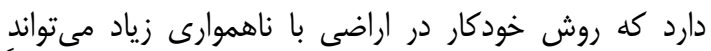

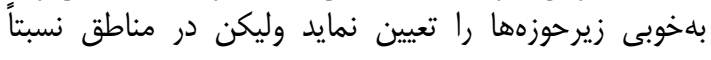
4- Illamanga
2- Geographic Resources Analysis Support System

5- Sediment Delivery Rate 
بتوان زيرحوزهها را در يك آبخيز با استفاده از داده رقومى نقي

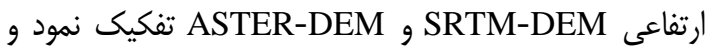

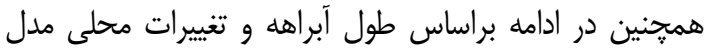

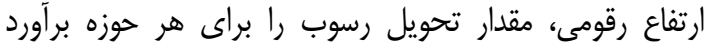

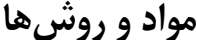

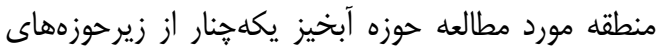

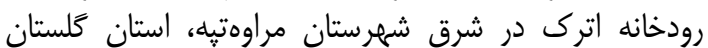

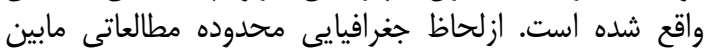

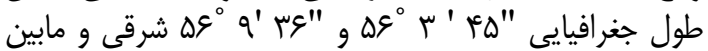

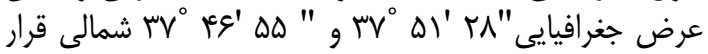

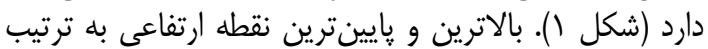

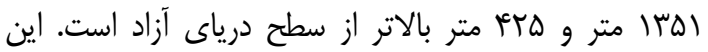
منطقه داراى سازند لسى است و توان رسوبز ايیى زيادى دارد.
و مزلقان واقع در استان مركزى شده است، نتايج تحقيق

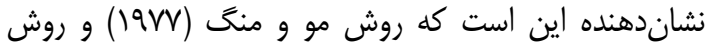

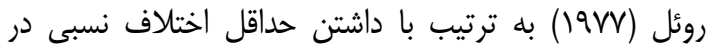

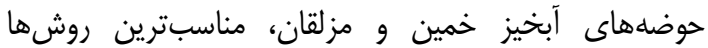

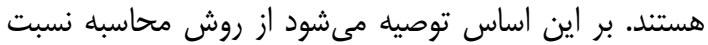

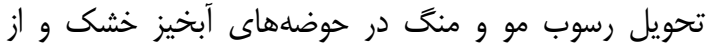

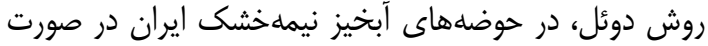

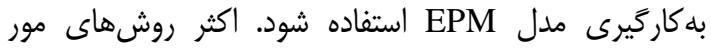

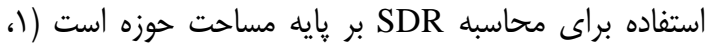

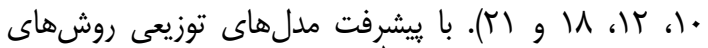

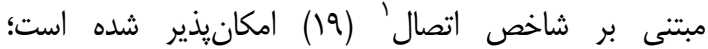
بdطورى كه مازول r.sdr در نرمافزار

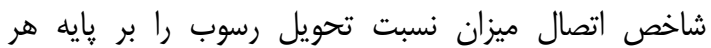
بيكسل برآورد مى كردد (\$). هدف از ائن اين تحقيق برآورد خودكار حوزهبندى است. بلهطورى كه با استفاده از نرمافزار

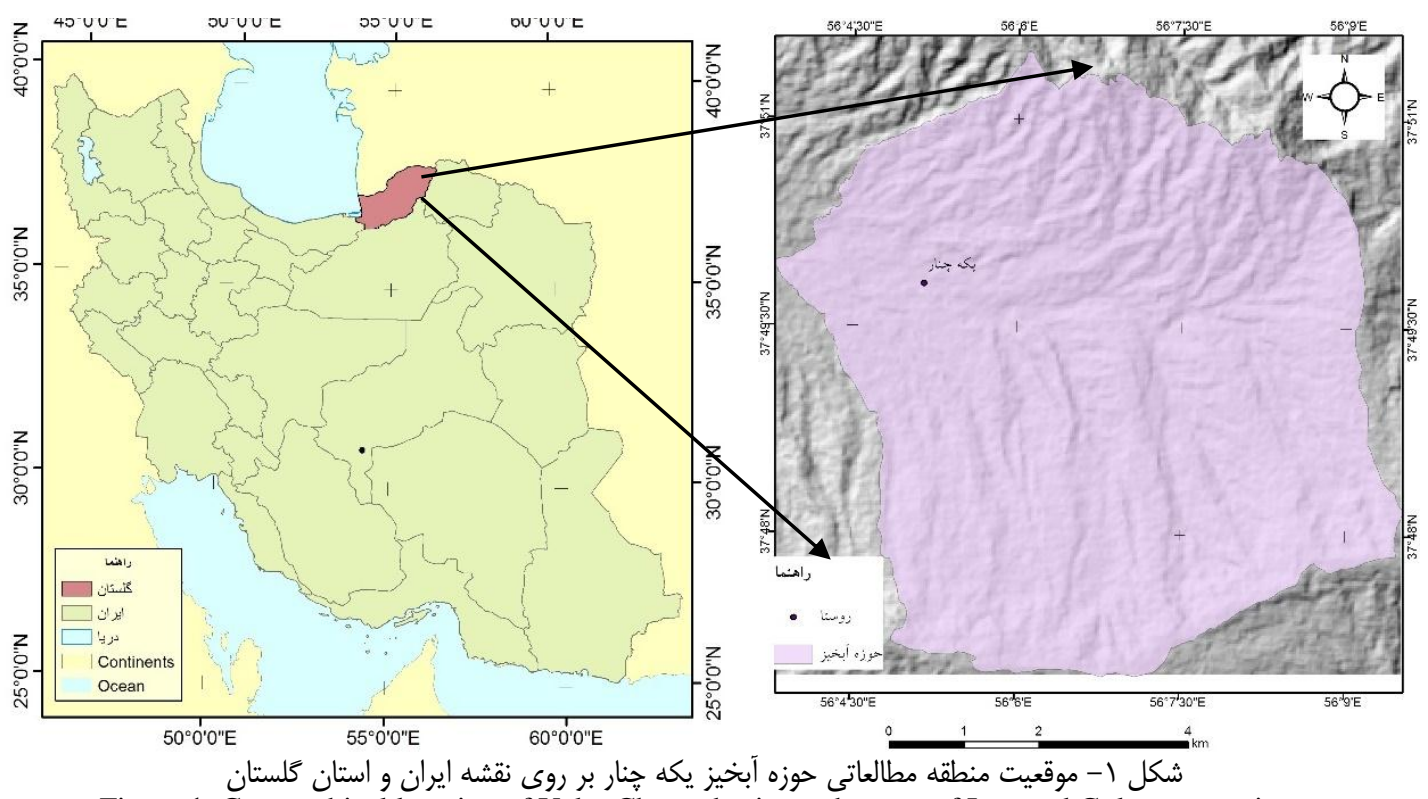

Figure 1. Geographical location of Yeke-Chenar basin on the map of Iran and Golestan province

$$
\text { موارد زير است (T): (ب) }
$$

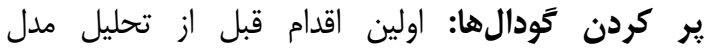

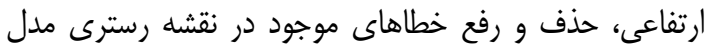

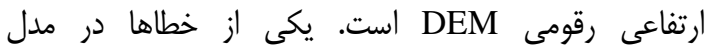

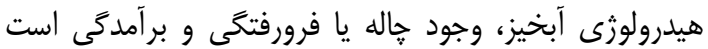

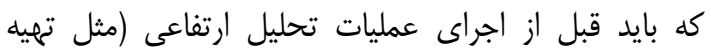

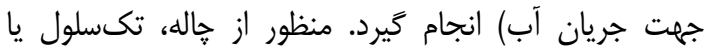

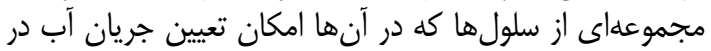

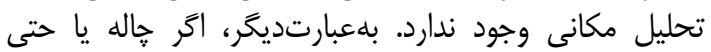

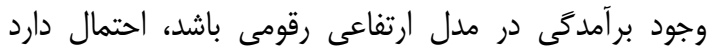

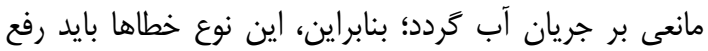

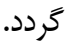

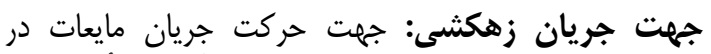

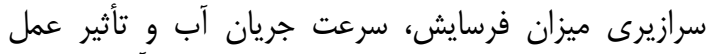
نيروى تخريبى بر ساختارهاى موجود در مسير آن آن را تعيين
نقشههاى مورد استفاده در اين تحقيق شامل مدل رقومى

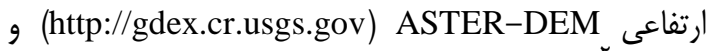

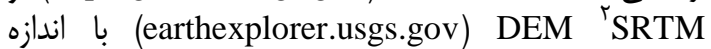

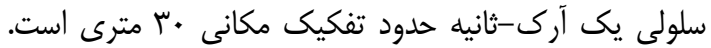

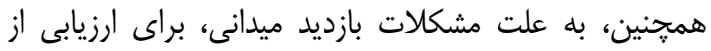

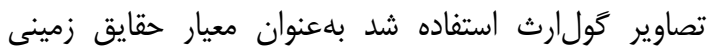

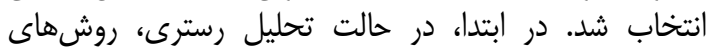

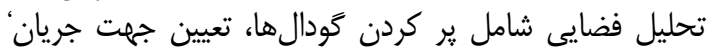

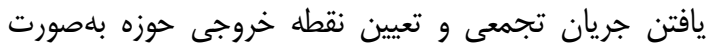

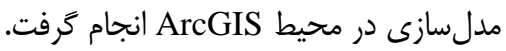

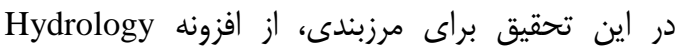

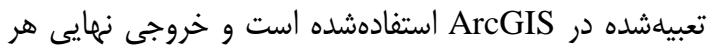

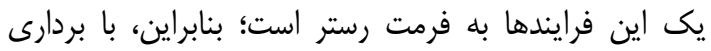

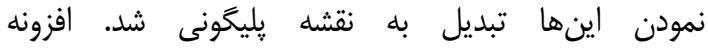
Hydrology 
منبع آبى دريافت مى كند. نقطه خروجى جريان، نقطهاى كهان

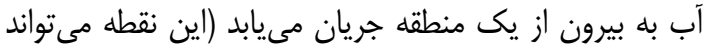

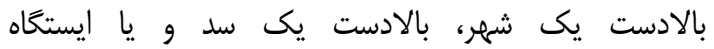
هيدرومترى باشد) كه براى تعيين مرز حوزه لازم است مشخص شود.

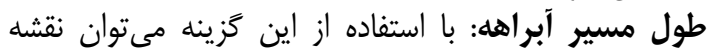

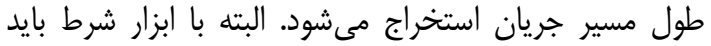

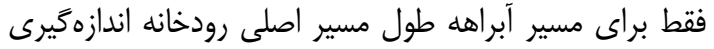

در اين تحقيق، در ابتدا نقشه مدل رقومى ارتفاع منطقه

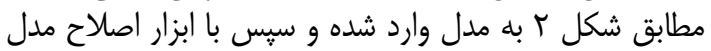

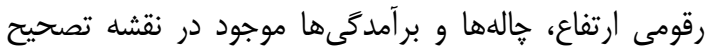

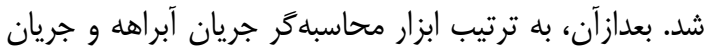

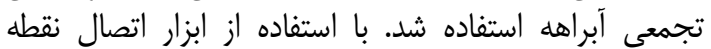

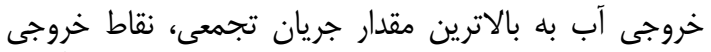

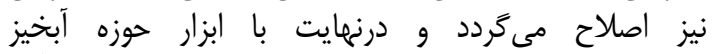

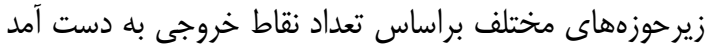

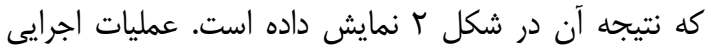

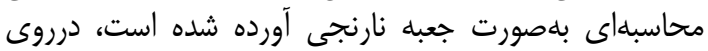

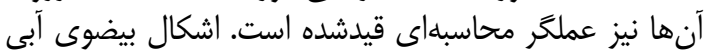

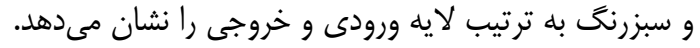

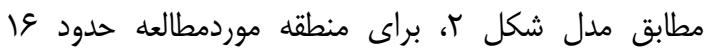

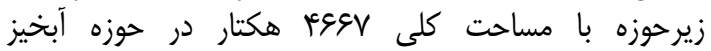

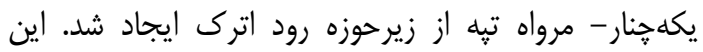

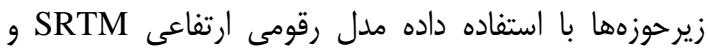

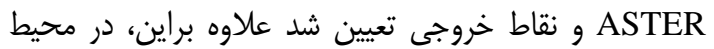

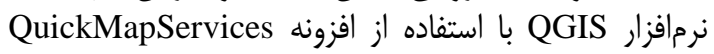

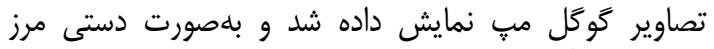

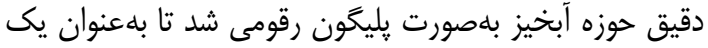

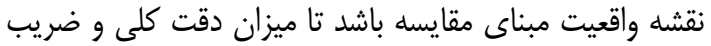

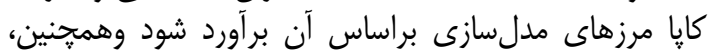

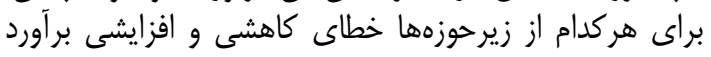

مىكند. وقتى آب در يكى سرازيرى بلهرف بإيين حركت

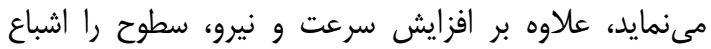

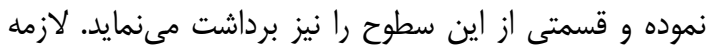

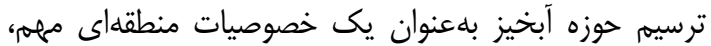

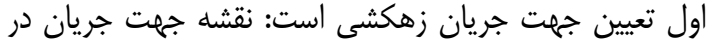

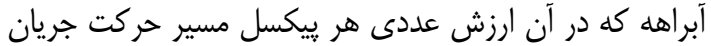

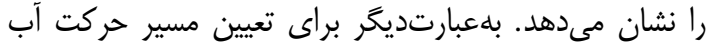

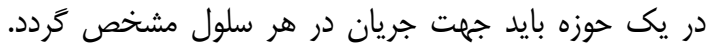

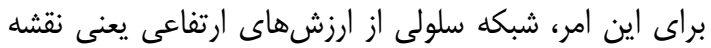

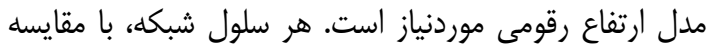

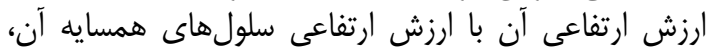

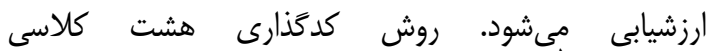

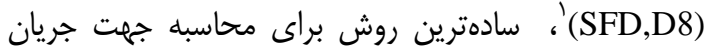

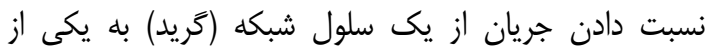

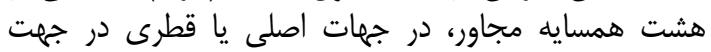

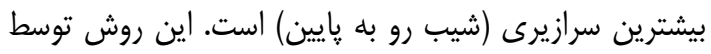

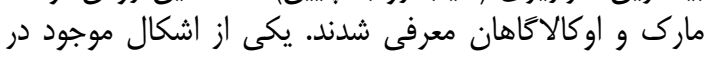

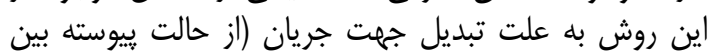

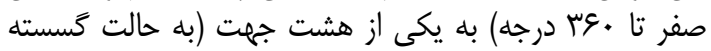

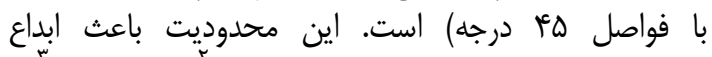

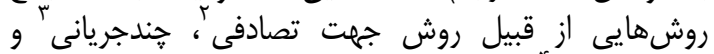

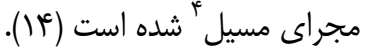

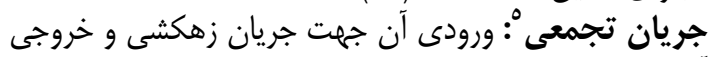

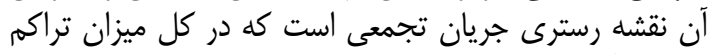

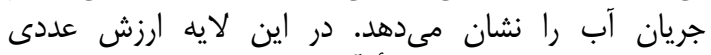

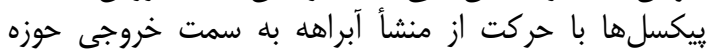

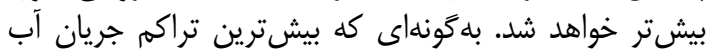

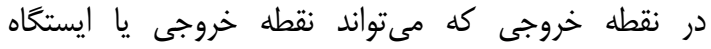

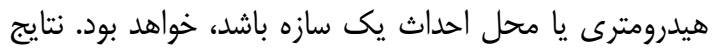

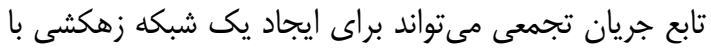

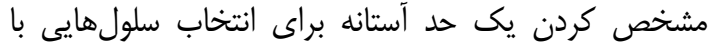

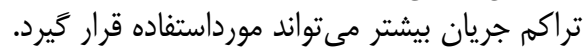

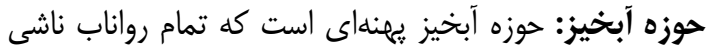

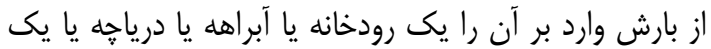




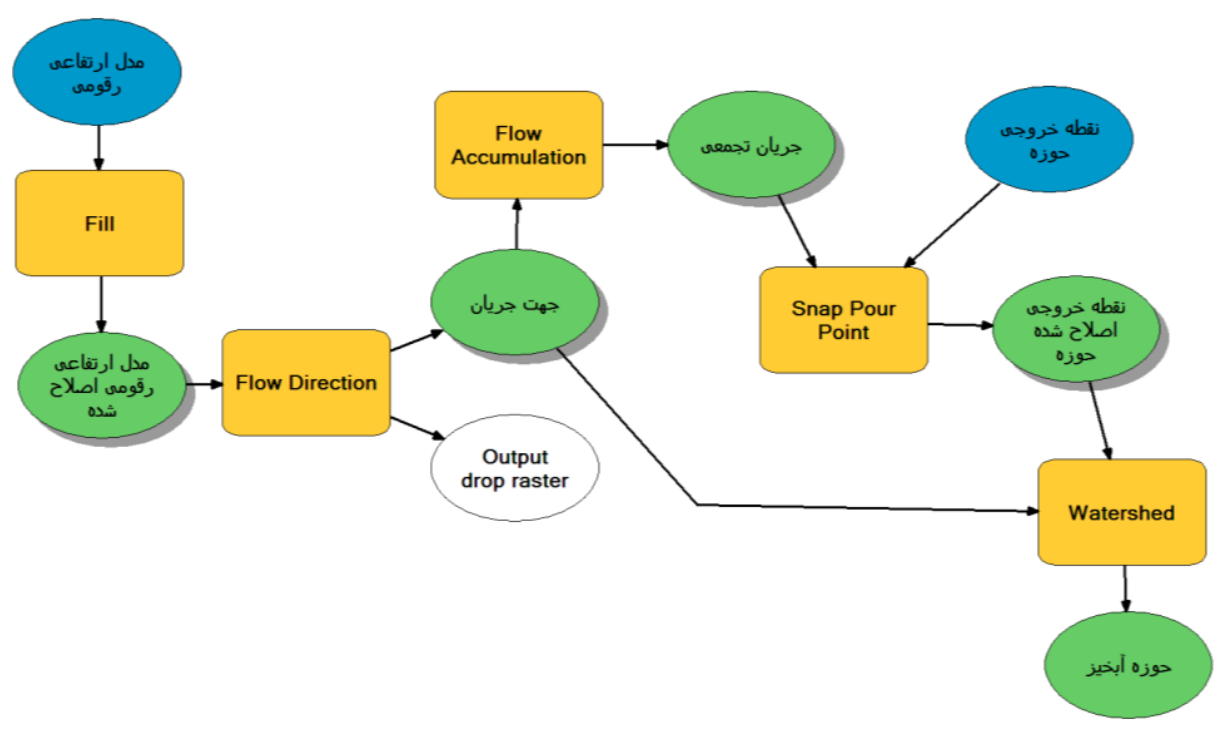

شكل r- مدل حوزه آبخيز در محيط نرمافزار ArcGIS (r)

Figure 2. Watershed model in ArcGIS and automated models (2)

كه هركدام شرايط خاص خود را دارا است، لازم به توضيح

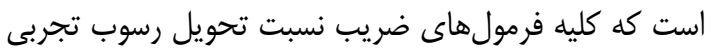

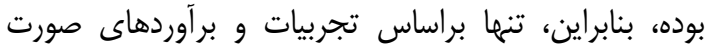

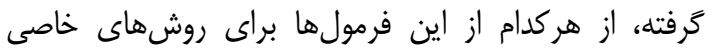

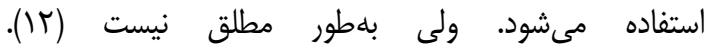

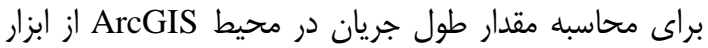
Flow Length

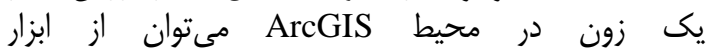
Zonal Statistics

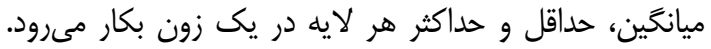

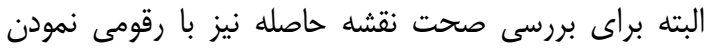

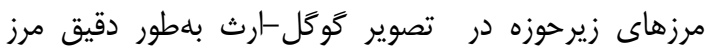

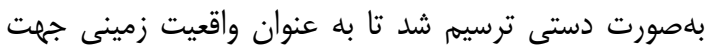

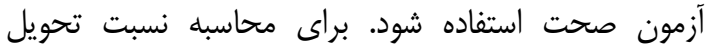

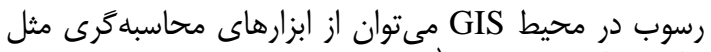

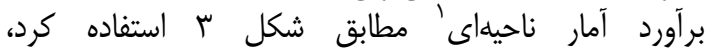

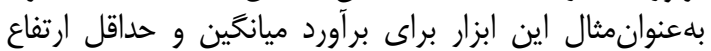
محلى براى هر حوزه بكار بردي

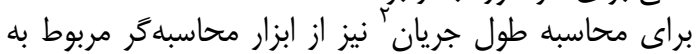

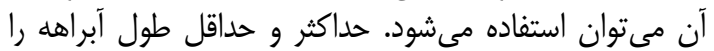

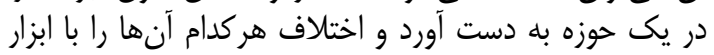

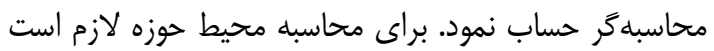

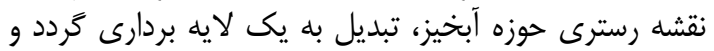

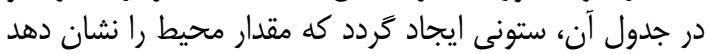

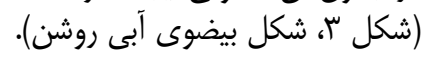

برآورد نسبت تحويل رسوب

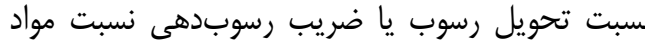

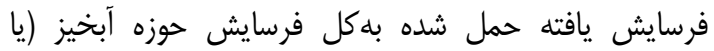

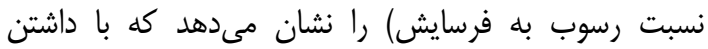

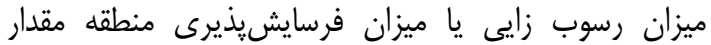

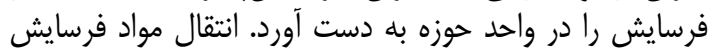

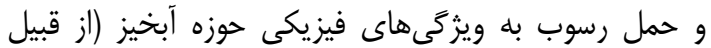

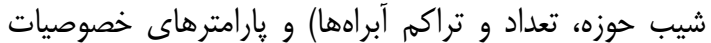

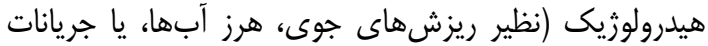

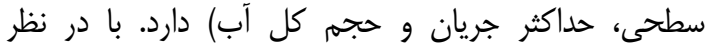

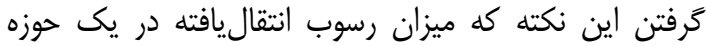

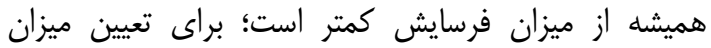

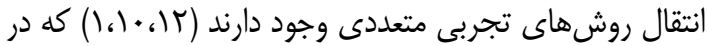

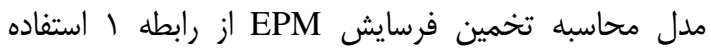

$R U=\frac{\sqrt{P \times D}}{L+10}$

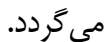
رابطه (1)

در رابطه فوق، RU، نسبت تحويل رسوب حوزه آبخيز را نشان

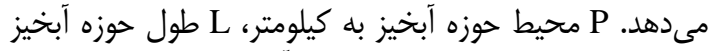

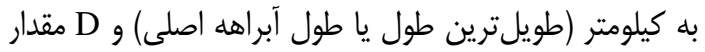

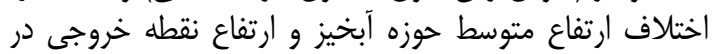
رودخانه اصلى است. با توجه به اينكه ميزان رسوب توليدى در يكى حوزه هميشه از

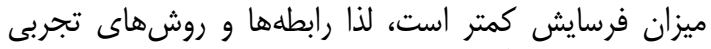

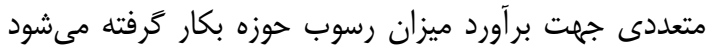




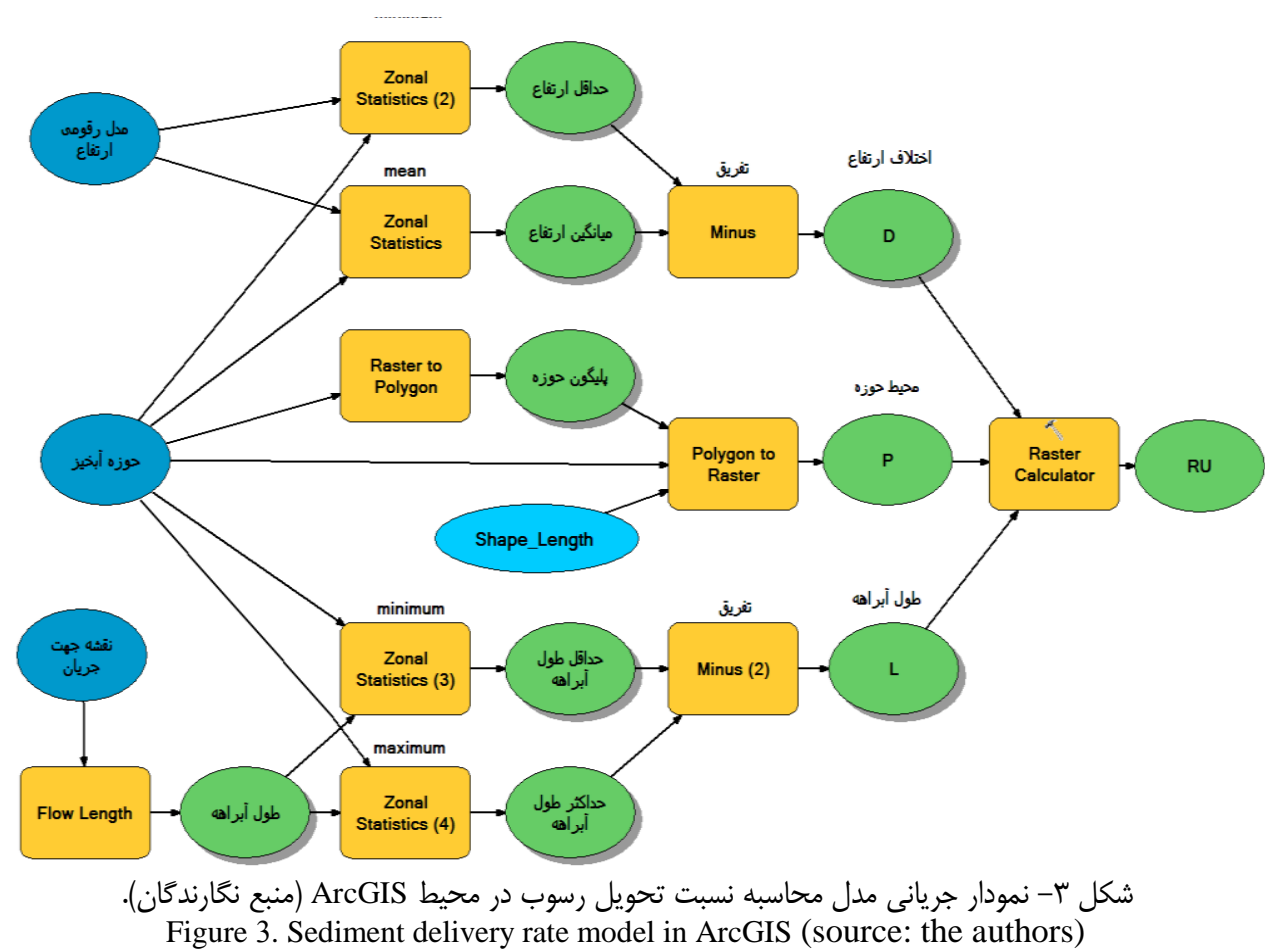

كلى و انطباق دو نقشه اسمى (كيفى) را نشان مىدهد واصد و اطمينان مىدهد دقت حاصله بلهُ دور تصنه اسمى (كادفى حاصل نشده است. $\mathrm{K}=\frac{P(A)-P(E)}{1-P(E)}$

(r) رابطه

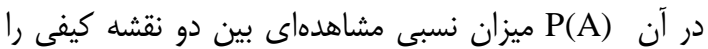

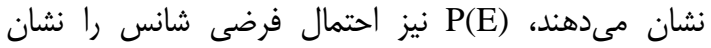

$$
\begin{aligned}
& P(A)=\frac{1}{n} \sum_{i-1}^{c} p_{i i} \\
& \text { رابطه (ب) } \\
& P(E)=\frac{1}{n^{2}} \sum_{i=1}^{c} p_{i T} p_{T j}
\end{aligned}
$$

در رابطه فوق pij سلول موجود در سطر i و ستون j است و

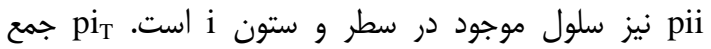

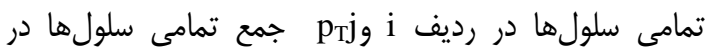

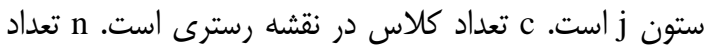

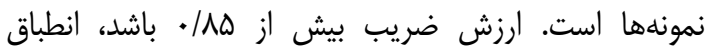

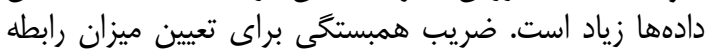

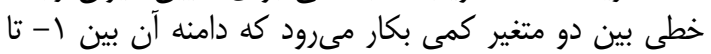
ا، به ترتيب رابطه منفى و رابطه مثلى بكار مثرد است:

$$
r=\frac{\sum(x-\bar{x})(y-\bar{y})}{\sqrt{\sum(x-\bar{x})^{2}(y-\bar{y})^{2}}}
$$

(ه) (ه)

در آن، r ميزان همبستخى (ييرسون)، X و y له به ترتيب متغير عددى اول و دوم و

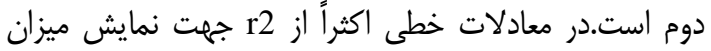
معنادارى رابطه خطى استفاده مى رَردا.
روش ارزيابى صحت مدل

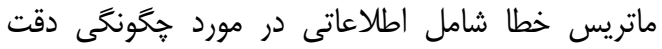

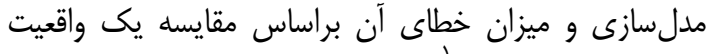

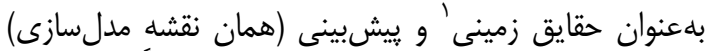

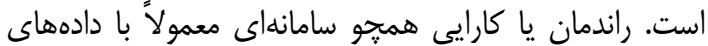

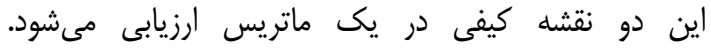

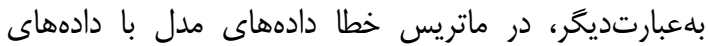

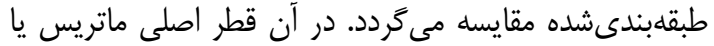

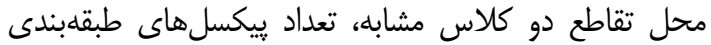

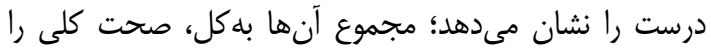

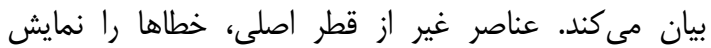

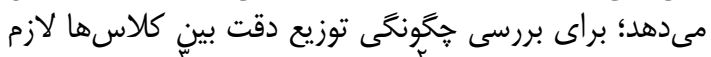

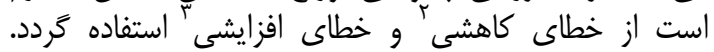

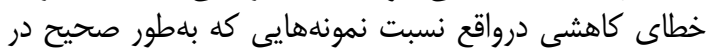

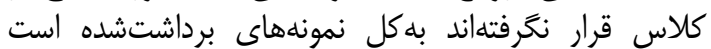

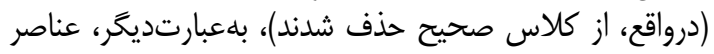

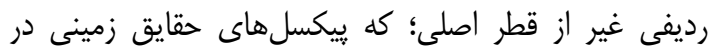

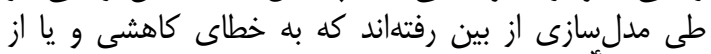

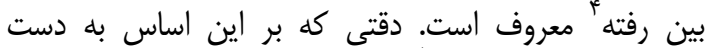

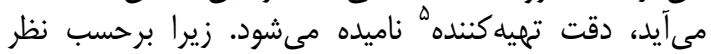

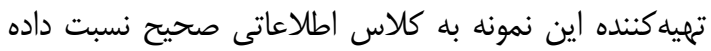

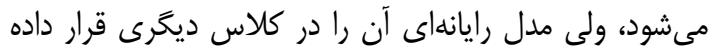

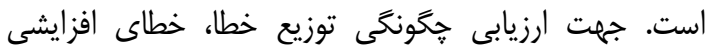

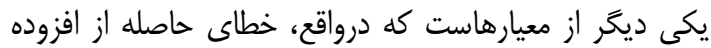

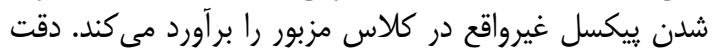

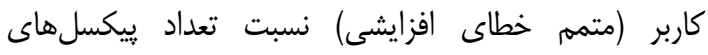

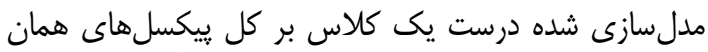

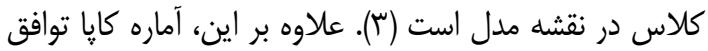


جدول ا، بيشترين خطاى كاهشى براى حوزهبندى مبتنى بر

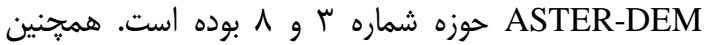

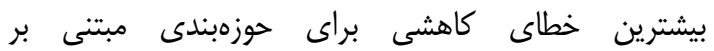
ASTER-DEM حوزه شماره سا است. به خاط خاطر اين كه اين

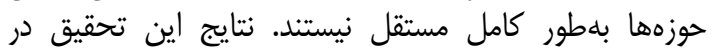

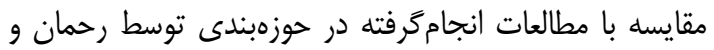

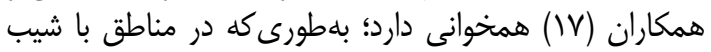

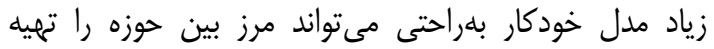

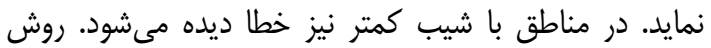

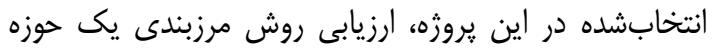

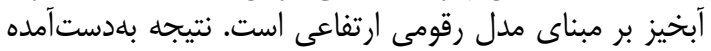

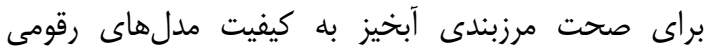

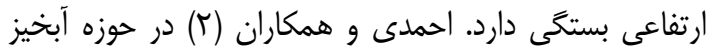

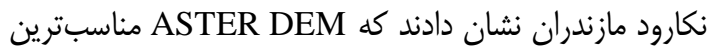

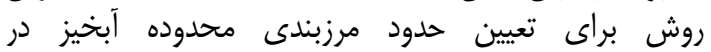

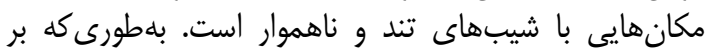

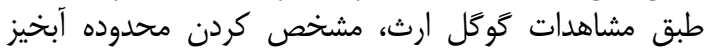

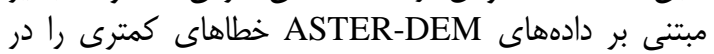
مقايسه با روش سنتى نشان داده است.

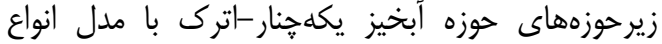
بحث و نتايج

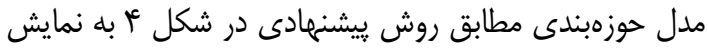

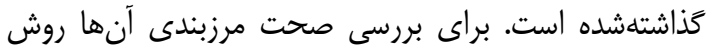

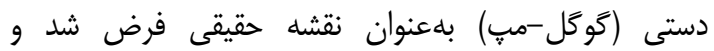

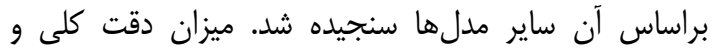

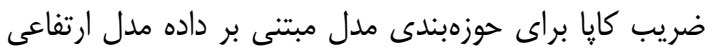
ASTER-DEM

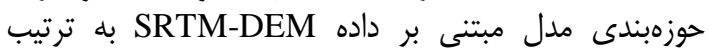

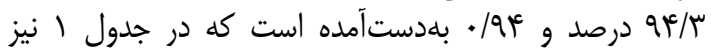

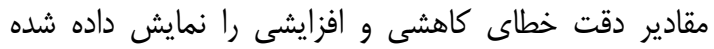

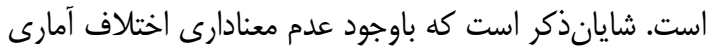

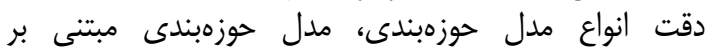

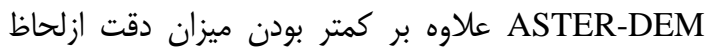

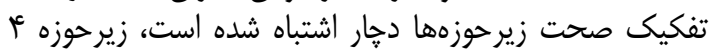

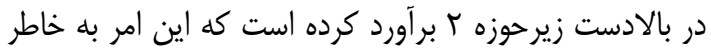

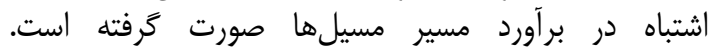
بلهطورى كه بدون تعيين نقطه خروجى براى خراى حوزه فوق، دقت

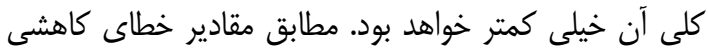

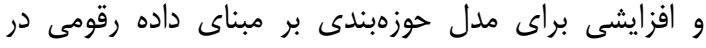

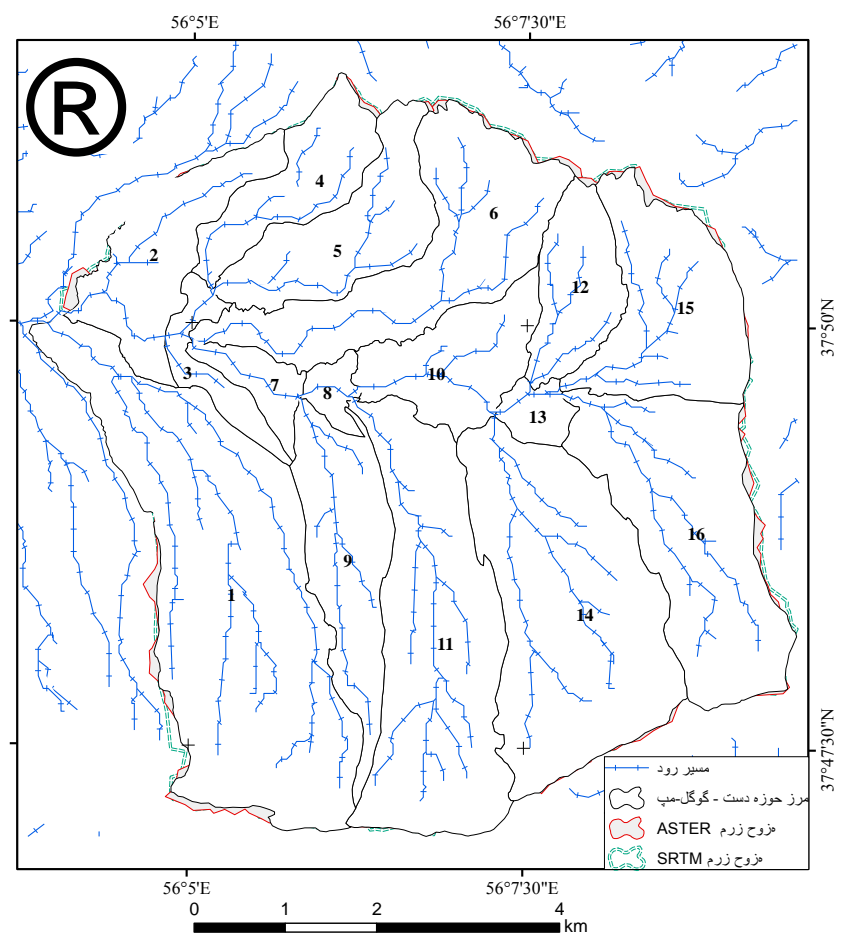

شكل أ- مرز حوزه آبخيز يكه جنار - اترك در محيط نرمافزار بهصورت روش دستى (گوكل -ارث) و مدل خودكار

Figure 4. The boundary of Yekechenar Watershed by manual method (based on Google Earth) and automated modeling 
جدول ا- مقادير خطاى كاهشى و افزايشى به درصد براى مدل حوزهبندى بر مبناى داده رقومى ارتفاعى ASTER و SRTM Table 1. The omission and commission errors for watershed delineation modeling based on ASTER-DEM and SRTM-DEM

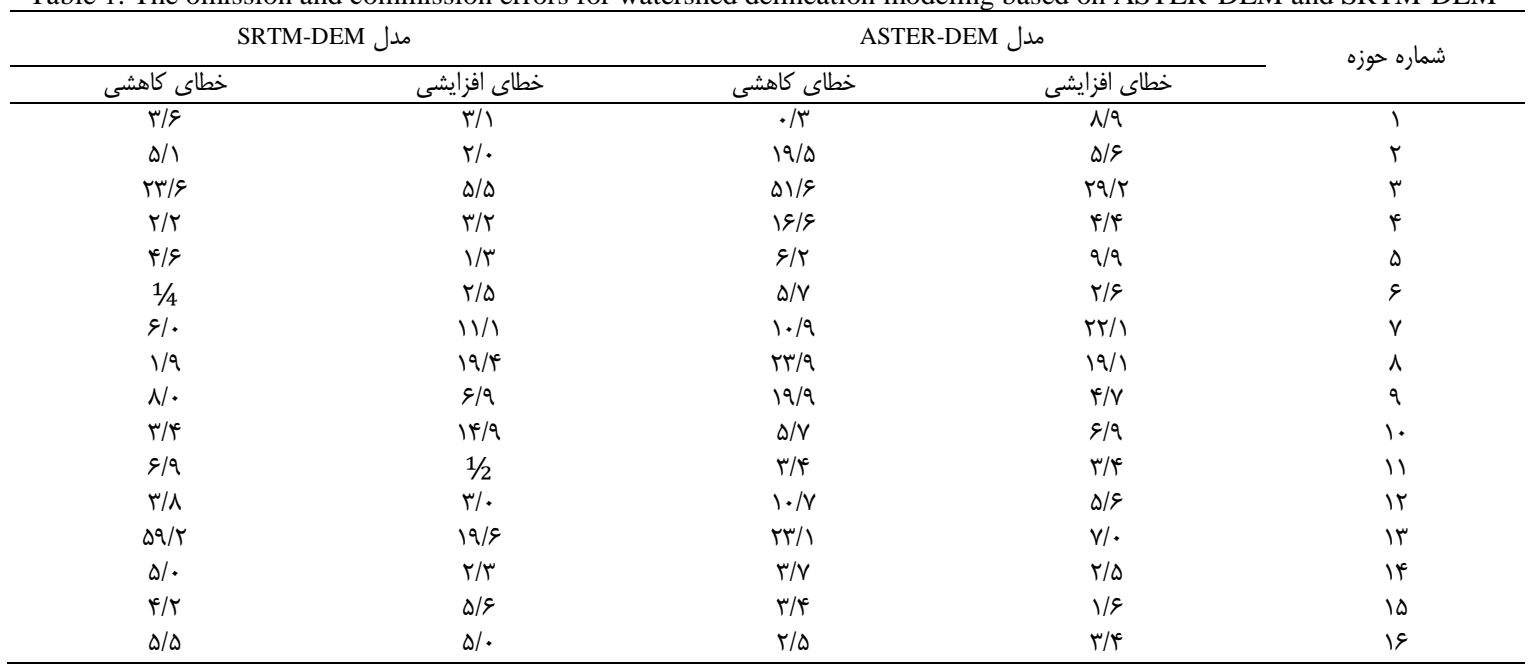

بهدستآمده از اين تحقيق مرزبندى حوزه آبخيز را با مقايسه

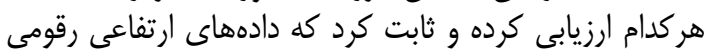

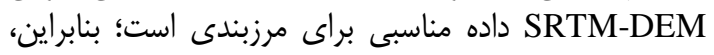

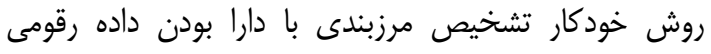

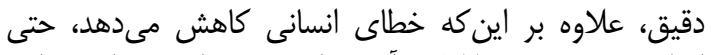

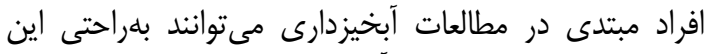
روش براى تعيين مرز حوزه آبخيز بكار ببرند.
در اين تحقيق نيز اختاف معنادارى بين دو روش مشاهده

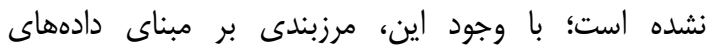

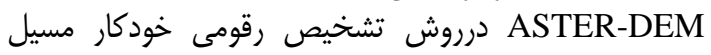

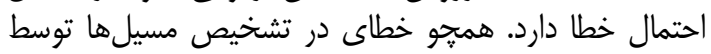

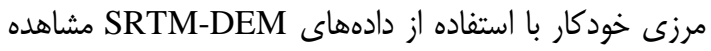

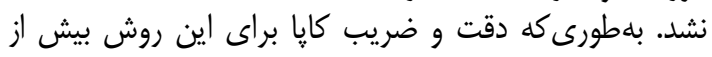

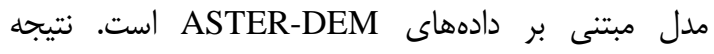

جدول r- ارزشهاى محيط حوزه P، طول مسير آبراه اصلى L و اختلاف ارتفاع محلى متوسط حوزه از نقطه خروجى D برحسب كيلومتر براى

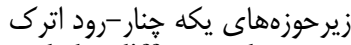

Table 2. The values of watershed perimeter P, the flow length of mainstream L and the difference between the mean elevation of watershed and outlet D in kilometers for sub-watersheds of Yekehchenar, the Atrak River

\begin{tabular}{|c|c|c|c|c|c|c|c|c|c|c|c|c|}
\hline \multicolumn{4}{|c|}{ مدل حوزه بندى دستى - گوكل -مي } & \multicolumn{4}{|c|}{ مدل حوزه بندى مبتنى بر SRTM-DEM } & \multicolumn{4}{|c|}{ مدل حوزه بندى مبتنى بر ASTER-DEM } & \multirow{2}{*}{ 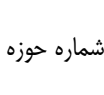 } \\
\hline $\mathrm{L}$ & $\mathrm{P}$ & $\mathrm{D}$ & RU & $\mathrm{L}$ & $\mathrm{P}$ & $\mathrm{D}$ & RU & $\mathrm{L}$ & $\mathrm{P}$ & $\mathrm{D}$ & RU & \\
\hline $\mathrm{V} / \Lambda$ & $11 / 9$ &.$/ \% \Delta$ & .148 & $V / \Delta$ & $10 / V$ & צ צא/. &.$/ D F$ & $V / \Delta$ & $18 / \mathrm{V}$ & g"א/. & $\cdot \mid \Delta S$ & 1 \\
\hline$r / \Lambda$ & $1 \% / \Delta$ & .11 & 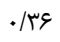 & $r / \cdot$ & $N / 9$ & $\cdot /$ & $\cdot / T \Lambda$ & $r /$ & N/q &.$/ 1$. &.$/ 49$ & r \\
\hline $1 / \cdot$ & १/. & $.1 \cdot 0$ &.$/ T \Delta$ & $\cdot / \Lambda$ & $\Delta / \Lambda$ & $\cdot / \cdot r^{c}$ & .119 & $\cdot / 1$ & $\varphi / V$ & $/ . r$ &.$/ 10$ & r \\
\hline$r / \mu$ & $V / r$ &.$/ 19$ &.$/ \mu$ & $r / r$ & $\mathrm{~V} / \mathrm{I}$ & $\cdot / r$ & • & $r / T$ & $s / \mathbb{C}^{4}$ &.$/ 19$ & $\cdot / \pi v$ & r \\
\hline$r / \kappa^{\epsilon}$ & $N / \Delta$ &.$/ r$. & . /\%q & $r / \mathcal{F}$ & $N / 1$ & $\cdot / r \cdot$ & . & $r / \kappa$ & $N / F$ &.$/ 19$ & . & $\Delta$ \\
\hline Q/द & $9 / 4$ & ع &.$/ 4$. & $\mathrm{Q} / \mathrm{S}$ & $\mid r / \Delta$ & ( &.$/ 48$ & $\Delta / \Gamma$ & $\mid r / 4$ & ع &.$/ 48$ & 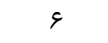 \\
\hline $1 / 4$ & $r / \cdot$ & $.1 \cdot \Delta$ & سו/. & $1 / 8$ & $\Delta / \cdot$ & $\cdot 1 \cdot \Delta$ &.$/ V V$ & $1 / 4$ & $r / V$ & $.1 . \Delta$ &.$/ 19$ & V \\
\hline$\cdot / \Lambda$ & $g / V$ & $\cdot 1 \cdot t^{c}$ &.$/ M$ & $\cdot / \mathrm{V} \Lambda$ & $\Gamma / \Delta$ & $\cdot 1 \cdot r^{c}$ &.$/ I^{F}$ & $\cdot / V$ & $ऍ /$. & .1 .4 &.$/ T$ & $\wedge$ \\
\hline$r / l$ & $11 /$ & $\cdot|r|$ & . & $r / \cdot$ & $1 . / \Delta F$ & T & $\cdot / 4 V$ & $r /$. & $\mathrm{V} / \mathrm{V}$ &.$/ I V$ & צr/. & 9 \\
\hline$r /$. & $r / r$ & .1 .9 &.$/ 1 \Lambda$ & $r / 1$ & $\Lambda / r$ & $\cdot /$ & ./rq & $r / 1$ & $\mathrm{~V} / \cdot$ & .1 .9 &.$/ T V$ & 1. \\
\hline$\Delta / \kappa^{\leftarrow}$ & $\mid r / \kappa$ & זس/. & $\cdot / \Delta \Delta$ & $\Delta / \Gamma$ & $\mid r / r$ & rא/. & $\cdot / \Delta T$ & $\Delta / \epsilon^{\epsilon}$ & $\mid r / 4$ & תז/. & $\cdot \mid \Delta F^{C}$ & 11 \\
\hline$r / 1$ & $9 /$. &.$/ M$ &.$/ \mu f^{c}$ & $r /$. & $\Delta / V$ &.$/ 1$ & אז/. & $r /$. & $\Delta / \Lambda$ &.$/ I V$ & זس/. & IT \\
\hline.$/ 9$ & $\kappa / \Delta$ & .1 .9 &.$/ M$ &.$/ 9$ & $r / \Lambda$ & $\cdot 1 \cdot e^{c}$ & . & $\cdot / V$ & $r / V$ & $.1 \cdot \Delta$ &.$/ 1 f^{c}$ & זו \\
\hline$r / r$ & $\mid \varepsilon / 8$ & $\cdot / T \Lambda$ &.$/ 8$. & $c / 1$ & $1 . / 9$ & $\cdot / T V$ &.$/ 49$ & $F / 1$ & $|r /|$ &.$/ T \Lambda$ & . $/ \Delta T$ & 14 \\
\hline$r / \Lambda$ & $V / V$ &.$/ I V$ & هـ/. & $r /$. & $N / \Lambda$ &.$/ I V$ & ג א/. & $r / 1$ & $\Lambda / \Lambda$ &.$/ 11$ & $\cdot / \mu$. & 10 \\
\hline$r / r$ & $11 \%$ & $\cdot / T V$ &.$/ 4 \lambda$ & $r / r$ & $1 . / \mu$ & . & . & $r / r$ & $11 / 1$ & צT/. &.$/ A^{q}$ & 19 \\
\hline
\end{tabular}


ترتيب س// • و و / • بهدست آمده است.

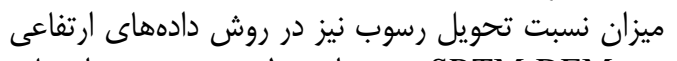

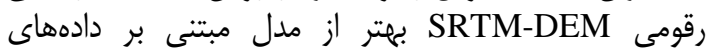

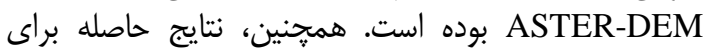

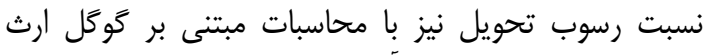

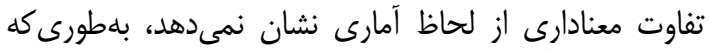

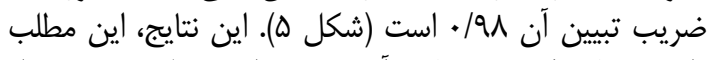

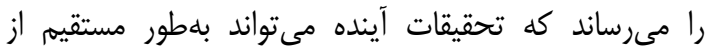

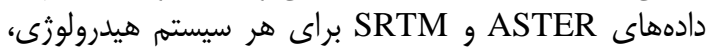

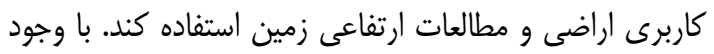

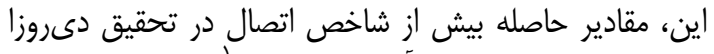
و همكاران (9) در حوزه آبخيز فيوكريك' - ايتاليا بوده است داست

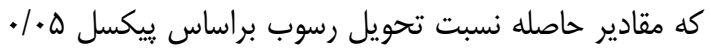
تا ه/• بوده است كه اين مربوط به خصوصيات زئورمولوزيكى

$$
\text { حوضه بستخى دارد. }
$$

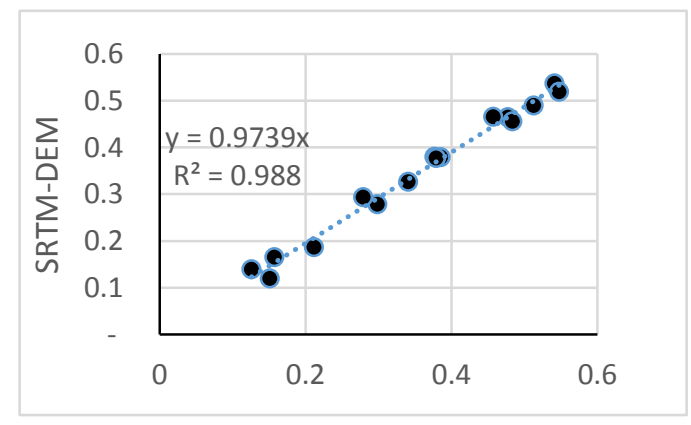

همجينين، نتايج محاسبه مدل نسبت تحويل رسوب براساس إس إسلا

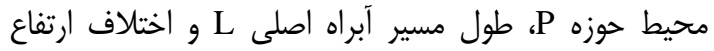

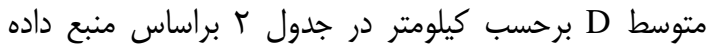

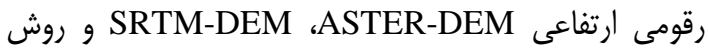

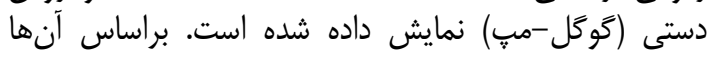

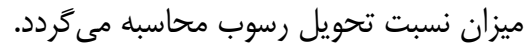

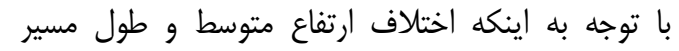

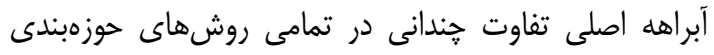

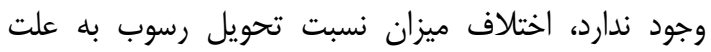

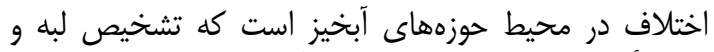

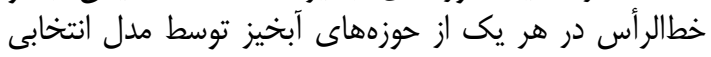

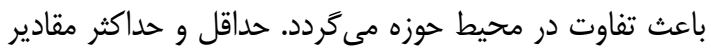

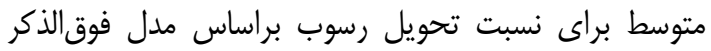

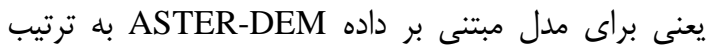

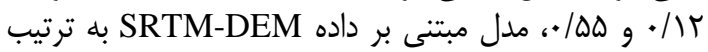

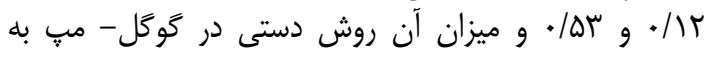

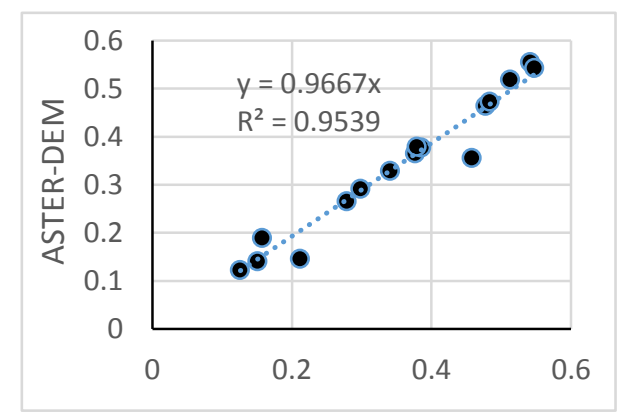

شكل ه- انطباق ميزان نسبت تحويل رسوب مدل خودكار (محور عمودى) با نسبت تحويل رسوب مدل دستى با استفاده از كوكل ارث (محور

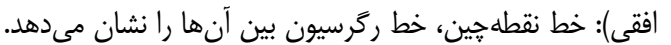

Figure 5. The correspondence of sediment delivery rate acquired using the automatic model (vertical axis) with manual model based on Google Earth (horizontal axis): the dotted line shows the regression line between them.

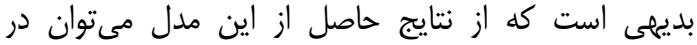

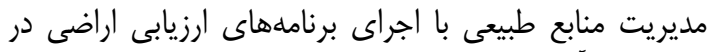

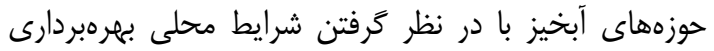

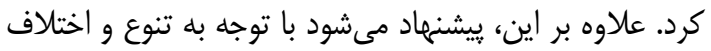

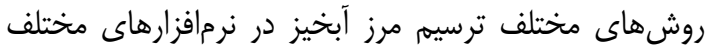
ارزيابى شود. - اري
شايانذكر است كه نتايج حاصل از اين تحقيق در تطبيق با

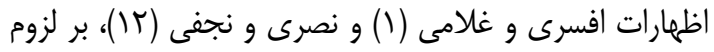

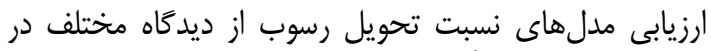

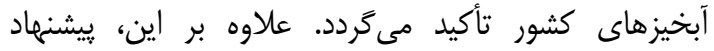

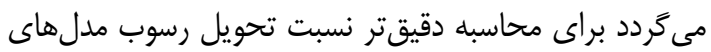
مكانمند و مدل توزيعى (థ) بكار برده شود. 
1. Afsari, R and J. Ghodousi. 2011. The Assessment of various methods of sediment delivery (SDR) in different climatic conditions (case study: watersheds of Markazi Province, Iran). Natural Geography, 4(12): 107-18 (In Persian).

2. Ahmadi, H., A. Das, M. Pourtaheri, C.B. Komaki and H. Khairy. 2014. Redefining the watershed line and stream networks via digital resources and topographic map using GIS and remote sensing (case study: the Neka River Watershed). Natural Hazards, 72(2): 711-722.

3. Alavipanah, S. K. and C.B. Komaki. 2007. The study of spectral separability information classes of lut desert using satellite data. Geography Researches, 38(3): 13-28 (In Persian).

4. Bera, A.K., V. Singh, N. Bankar, S. S. Salunkhe and J.R. Sharma. 2013. Watershed delineation in Flat Terrain of Thar Desert Region in North West India - a semi-automated approach using DEM. Journal of the Indian Society of Remote Sensing, 41(1): 187-199.

5. Chang, C.L. 2009. The impact of watershed delineation on hydrology and water quality simulation. Environmental Monitoring and Assessment, 148(1-4): 159-65.

6. De Rosa, P., C. Cencetti and A. Fredduzzi. 2016. A GRASS Tool for the Sediment Delivery Ratio Mapping. PeerJ Preprints 4, https://doi.org/10.7287/peerj.preprints.2227v2.

7. DeMers, Michael N. 2009. GIS for Dummies. Hoboken, NJ: Wiley. 348 pp.

8. Gopinath, G., T.V. Swetha and M.K. Ashitha. 2014. Automated extraction of watershed boundary and drainage network from SRTM and comparison with survey of India Toposheet. Arabian Journal of Geosciences, 7(7): 2625-2632.

9. Haralick, R.M. and G.L. Kelly. 1969. Pattern recognition with measurement space and spatial clustering for multiple images. Proceedings of the IEEE, 57(4): 654-65.

10. Khosravi, K., A. Safari. M. Habibnejad Roshan and N. Mahmoudi. 2012. Evaluation of soil erosion and sediment yield estimation various empirical model by observation values (Case Study: Babolroud Watershed, Mazandaran Province). Environmental Erosion Researches, 1(4): 33-53 (In Persian).

11. Lockhart, J.J. 1991. A comparison of manual and automated methods for delimiting watersheds for use with GRASS/GIS software. DTIC Document. USACERL Technical Report N-91/34. 1-31pp. US US Army Corps of Engineers, Construction Engineering Research Laboratory.

12. Nasri, M. and A. Najafi. 2015. Determining of mathematical relationships sediment delivery rate and watershed factors. Natural Ecosystems of Iran, 6(2): 1-12 (In Persian).

13. O'Banion, R., I. Alameddine, A. Gronewold and K. Reckhow. 2008. PyLIDEM: A Python-Based Tool to Delineate Coastal Watersheds Using LIDAR Data. AGU Fall Meeting Abstracts 1 (December): San Francisco, CA.

14. O'Callaghan, J.F. and D.M. Mark. 1984. The extraction of drainage networks from digital elevation data. Computer Vision, Graphics, and Image Processing, 28(3): 323-344.

15. Pratt, W.K. 2007. Digital Image Processing PIKS Scientific Inside. 4th ed. Hoboken, NJ: Wiley. 812 pp. Wiley-Interscience. Hoboken, N.J.

16. Pryde, J.K., J. Osorio, M.L. Wolfe, C. Heatwole, B. Benham and A. Cardenas. 2016. Comparison of watershed boundaries derived from SRTM and ASTER digital elevation datasets and from a digitized topographic map. 1-10 pp. An ASABE Meeting Presentation, Minnesota, USA.

17. Rahman, M.M., D.S. Arya and N.K. Goel. 2010. Limitation of $90 \mathrm{~m}$ SRTM DEM in drainage network delineation using D8 Method - a case study in Flat Terrain of Bangladesh. Applied Geomatics, 2(2): 49-58.

18. Rostami, N. 2009. Selection of Best Model of SDR estimation in Illam dam. M.Sc. Thesis, Keraj: Tehran University (In Persian).

19. Vigiak, O., L. Borselli, L.T.H. Newham, J. McInnes and A.M. Roberts. 2012. Comparison of conceptual landscape metrics to define hillslope-scale sediment delivery ratio. Geomorphology, 138(1): 74-88.

20. Wang, Yu-Hsiang. 2010. Tutorial: Image Segmentation. National Taiwan University, Taipei, 1-36.

21.Zhou, W. and W.U. Bingfang. 2008. Assessment of soil erosion and sediment delivery ratio using remote sensing and GIS: A case study of Upstream Chaobaihe River Catchment, North China. International Journal of Sediment Research, 23(2): 167-73. 


\title{
Comparison of Automatic Extraction of Sediment Delivery of Watershed and Traditional Method in Geographic Information System (Case Study: Yekechenar Watershed -Golestan Province)
}

\section{Chooghi Bairam Komaki ${ }^{1}$, Hassan Ahmadi', Maryam Mombini ${ }^{3}$, Sajjad Ahmad Yousefi ${ }^{4}$ and Naser Mostafavi $^{5}$}

\author{
1- Assistant Professor, Department of Arid Regions Management, Gorgan University of Agricultural Sciences and \\ Natural Resources, (Corresponding Author: bkomaki@ gmail.com). \\ 2- Assistant Professor, Humanities Faculty, Tarbiat Modares University \\ 3- PhD Student of Desertification Combat, Department of Arid Regions Management Gorgan University of \\ Agricultural Sciences and Natural Resources \\ 4- PhD Student of Water and Soil Protection, Department of Watershed Management, Gorgan University of \\ Agricultural Sciences and Natural Resources \\ 5- PhD Student of Management and Control of Desert, Department of Natural Resources, Tehran University \\ Received: April 25, 2017 \\ Accepted: June 12, 2017
}

\begin{abstract}
Determining the sediment delivery rate and watershed delineation is among the first stage in environmental research, especially in water erosion estimation. The determining the accurate boundary of watershed is important for the hydrological and morphologic characteristics of watersheds. The study aims to present an automatic extraction model of watershed delineation and calculate sediment delivery rate. The traditional method of determining the watershed delineation, and subsequently, calculating sediment delivery rate is performed manually that using a topographic map, the boundary of the watershed is determined and main flow length is calculated. However, nowadays due to the advancement of digital analytical spatial-based methods in GIS software, automatic delineation of watershed is feasible. For this purpose, the errors of digital elevation model are removed. After calculating the flow direction, and flow accumulation, watershed boundaries can be determined having pourpoints. Then, sediment delivery rate is calculated by local height of watershed, its perimeter, and flow length. In this research, the digital elevation models of ASTER-DEM and SRTM-DEM are utilized to design watershed delineation model and to evaluate the overall accuracy and the correspondence of them, so the border of watershed is delineated using Google map, which is used as a ground truth. The findings of this study show the automatic extraction of watershed boundary and calculation of sediment delivery rate do not have significant differences with traditional mothed. So that, the overall accuracy and Kappa index of the watershed boundary based on ASTERDEM are 93 percent and 0.92, respectively, and their values for the watershed boundary based on SRTM-DEM are 94.3 percent and 0.94 , respectively. The correlation coefficient $\left(\mathrm{r}^{2}\right)$ of calculated sediment delivery rate based on SRTM-DEM is 0.98 and its value based on ASTERDEM is 0.95 .
\end{abstract}

Keywords: Watershed Delineation, Digital Elevation Model, Sediment Delivery Rate, Atrak River 